\title{
The diagnostic value of evaluating the maximum velocity of blood flow in the uterine arteries of women with postmenopausal bleeding
}

\author{
Dariusz Samulak • Maciej Wilczak • \\ Monika Englert-Golon · Magdalena Monika Michalska
}

Received: 9 August 2010/ Accepted: 16 December 2010/Published online: 1 January 2011

(C) The Author(s) 2010. This article is published with open access at Springerlink.com

\begin{abstract}
Purpose The aim was to evaluate the utility of ultrasonographic examinations, such as the Doppler technique, in diagnosing women with postmenopausal bleeding.

Methods Specifically, maximum end-diastolic velocity of blood flow (MEDV), time-averaged maximum velocity of blood flow (TAMXV) and peak systolic velocity of blood flow (PSV) were evaluated. Data were obtained and analyzed from a group of 100 female patients diagnosed and treated because of abnormal bleeding from the genitals in the Gynecological-Obstetrics Clinical Hospital of Poznan University of Medical Sciences. The following packages were used for statistic analyses: STATISTICA v 7.1 (StatSoft, Inc. 2005), StatXACT v.5.0.3, CYTEL SOFTWARE CORPORATION and Analyse-it Software v.1.68. Results The parameters evaluated were highest in the carcinoma group, lower when proliferation was diagnosed and the lowest in the control group.
\end{abstract}

D. Samulak $(\bowtie) \cdot$ M. M. Michalska

Department of Obstetrics and Gynecology,

Regional Hospital in Kalisz, ul. Toruńska 7,

62-800 Kalisz, Poland

e-mail: samulakd@wp.pl

D. Samulak

Clinic of Mother's and Child's Health,

Poznan University of Medical Sciences, Poznan, Poland

D. Samulak · M. Englert-Golon

Clinic of Gynecological Surgery,

Poznan University of Medical Sciences, Poznan, Poland

M. Wilczak

Department of Medical Education,

Poznan University of Medical Sciences, Poznan, Poland
Conclusions Transvaginal ultrasonography diagnostics using the Doppler technique was found to play an important role in the diagnostic process of pathologies within the endometrium.

Keywords Endometrial carcinoma - MEDV .

Proliferation of the endometrium · PSV · TAMXV

\section{Introduction}

The introduction of ultrasonography to gynecological diagnostics has improved the precision with which the endometrium can be evaluated in physiological and pathological states. Although carcinoma of the endometrium does not show typical ultrasonographic features, ultrasonographic examination with a transvaginal probe is typically used to detect these neoplasms. This examination is also very useful in evaluating the degree of infiltration before an operation. Ultrasonography is non-invasive and is currently one of the most frequently used diagnostic techniques for evaluating the endometrium. Thus, ultrasonography has become an important supplement to a routine gynecological examination, and clinical procedures are often based on the results of this examination. However, using this technique to measure the thickness of the endometrium itself is not sufficient [1-4].

Imaging using ultrasonography with the color Doppler technique created the potential to distinguish malignant changes from non-malignant changes. This is one of the most advanced diagnostic methods used in the diagnostic process of female genitals. Analyses of blood flowvelocity in artery vessels based on maximum blood flowvelocities makes it possible to create objective Doppler measurements, which allows for comparisons using any 
apparatus. However, due to existing controversies related to the measurement of particular Doppler parameters, the aim of this paper was to analyze the perfusion in uterine arteries in women hospitalized due to abnormal bleeding from the uterus using vaginal ultrasonography with color Doppler.

\section{Materials and methods}

A group of 100 female patients diagnosed with and treated for abnormal bleeding from the uterus in the Clinic of Gynecological Surgery of the Gynecological-Obstetrics Clinical Hospital of Poznan University of Medical Sciences between December 2005 and January 2007, were studied. The median age of endometrial cancer patients was 62 years, of patients with endometrial hyperplasia was 59 years and 46 years for the women without endometrial pathology.

Gynecological examination and ultrasounds were always done by the same doctor. During ultrasound examination with empty bladder of patients we used vaginal probe 5.0-6.5 Hz of Aloka model 5500. After analysis in the gray scale we always performed Doppler examination analysis.

Based on the analysis of blood velocity wave form in artery vessels, the following parameters were evaluated: time-averaged maximum velocity of blood flow (TAM$\mathrm{XV}$ ), maximum end-diastolic velocity of blood flow (MEDV) and peak systolic velocity of blood flow (PSV). These values were calculated automatically using ultrasonographic software. Average values of three measurements of consecutive blood velocity waves were assumed to be representative for the examination conducted. Results of this ultrasonography examination were compared with a histopathological examination of the endometrium. To evaluate the endometrium, the diagnostic curettage was done and in some cases the endometrium was collected after hysterectomy. Patients were divided into following groups: 43 patients in whom endometrial carcinoma was diagnosed during further diagnostics; 11 women in whom hyperplastic changes of endometrium were detected during further diagnostics and 46 patients in whom no changes were diagnosed in the endometrium, as a control group. Histopathological examination was performed in histopathological laboratory in our hospital.

Curettage was always performed in general anesthesia, after dilatation of cervical canal. We collected material from the cervix and from the uterine cavity in different glasses and after the procedure the material was sent immediately to the histopathological laboratory. Hysterectomies were always performed transabdominaly in the general anesthesia.
The results obtained were submitted to a statistical analysis. TAMXV, MEDV and PSV values, measured on an interval scale due to the diagonal distribution of the parameters analyzed, were characterized with the aid of median values. The distributions of the above-mentioned parameters were assessed to determine their normality using a Shapiro-Wilk test. An analysis of variance ANOVA with a Newman-Keuls post-hoc test and a nonparametric Kruskal-Wallis test with Dunn's multiple comparisons test were used in order to make comparisons between the three groups. A level of 0.05 was assumed for the risk of erroneous conclusions and for the significance level in this paper.

Calculations were performed using the STATISTICA v 7.1 (StatSoft, Inc. 2005) statistical package, as well as StatXACT v.5.0.3 by CYTEL SOFTWARE CORPORATION and Analyse-it Software v.1.68.

The studies have been approved by The Ethics Committee in the University of Medical Sciences in Poznan on 8 December 2005 and the project number is $1681 / 05$.

\section{Results}

After histopathological examination we observed three groups of patients: 43 patients with endometrial carcinoma, 11 women with hyperplastic changes of the endometrium and 46 patients with no pathological changes in the endometrium, as a control group.

After statistical analysis it was shown that endometrial hyperplasia and carcinoma are found most frequently in urban women aged 51-60 years old. Among patients living in the country these lesions were observed much less frequently. Before the age of 40 endometrial carcinoma was found occasionally. It turned out that among factors coinciding with these endometrial pathologies the biggest importance was found for obesity, arterial hypertension and diabetes. Endometrial hyperplasia was most frequently accompanied by cholecystolithiasis, while the risk of endometrial carcinomas was much less affected by this disease. Menopause after the age of 51 years increased the risk of development of carcinoma or hyperplasia. However, early menarche was not recorded in these groups. Increased parity was not a protective factor against the disease.

Relationships occurring between the three groups of patients and the TAMXV are shown in Table 1.

The highest median value of TAMXV, $18.50 \mathrm{~cm} / \mathrm{s}$, was observed in the group with carcinoma of the endometrium. The median value of TAMXV was lower in the group of proliferations $(\mathrm{TAMXV}=17.00 \mathrm{~cm} / \mathrm{s}$ ) and the lowest in the control group $(16.40 \mathrm{~cm} / \mathrm{s})$.

When comparing average values of TAMXV in the different groups, higher values were observed in patients 
Table 1 Comparison of TAMXV $(\mathrm{cm} / \mathrm{s})$ values in the cancer and proliferation groups and in the control group

\begin{tabular}{lccccccc}
\hline & $\begin{array}{l}\text { Number } \\
\text { of patients }\end{array}$ & Average & Minimum & Maximum & Median & 25 percentile & 75 percentile \\
\hline Carcinoma & 43 & 20.19070 & 10.20000 & 36.90000 & 18.50000 & 14.70000 & 24.60000 \\
Proliferation & 11 & 18.49091 & 12.60000 & 28.50000 & 17.00000 & 15.30000 & 22.40000 \\
Control group & 46 & 17.20000 & 9.60000 & 28.60000 & 16.40000 & 13.70000 & 21.10000 \\
Total & 100 & 18.62800 & 9.60000 & 36.90000 & 17.20000 & 14.20000 & 22.40000 \\
$P$ value & & $>0.05$ & $>0.05$ & $>0.05$ & $>0.05$ & $>0.05$ & $>0.05$ \\
\hline
\end{tabular}

with carcinoma (TAMXV $=20.19 \mathrm{~cm} / \mathrm{s}$ ) and with proliferation of the endometrium $($ TAMXV $=18.49 \mathrm{~cm} / \mathrm{s}$ ) than in women with no pathology within the endometrium (TAMXV $=17.20 \mathrm{~cm} / \mathrm{s})$. However, these differences were not statistically significant. MEDV values based on measurements in uterine arteries are shown in Table 2.

The median value of MEDV was $10.00 \mathrm{~cm} / \mathrm{s}$ in patients with carcinoma, $8.90 \mathrm{~cm} / \mathrm{s}$ in patients with proliferation and $7.95 \mathrm{~cm} / \mathrm{s}$ in the control group. Average MEDV values were lowest in the group with carcinoma of the endometrium, amounting to $10.60 \mathrm{~cm} / \mathrm{s}$. Similar results were obtained in the group of proliferations (MEDV $=8.58 \mathrm{~cm} / \mathrm{s}$ ) and in the control group (MEDV $=8.99 \mathrm{~cm} / \mathrm{s})$. However, no significant differences of MEDV were found in the statistical analyses performed.

In the carcinoma and proliferation groups, similar median values of PSV, 34.10 and $34.20 \mathrm{~cm} / \mathrm{s}$, were obtained. A lower median value, $29.55 \mathrm{~cm} / \mathrm{s}$, was obtained in the control group. Comparisons of average values of maximum systolic velocity of blood flow did not show any statistically significant differences between the three groups, despite the fact that the PSV values obtained were highest in women with carcinoma, lower in patients with proliferation and much lower in the control group (Table 3).

To summarize the results of the analysis, the highest values of TAMXV, MEDV and PSV were observed in the carcinoma group, while values of these parameters were lower in the proliferation group and lowest in the control group. However, it is important to highlight that no parameter measured was significantly higher in the group of patients with carcinoma or proliferation in comparison with the control group.

\section{Discussion}

In recent years, many studies have focused on the use of the Doppler technique in evaluating blood flow in artery vessels. Many authors have reported that these examinations are important in enabling non-malignant and malignant changes to be distinguished. In the results of the present study, no statistically significant increase of maximum blood flow velocity in uterine arteries was observed in patients with carcinoma or proliferation in comparison to the group showing no pathology within the endometrium. Our results are compatible with the results obtained by Sladkevicius et al. [5]. This study found no statistically significant differences between TAMXV values in groups of women studied in an analysis of blood perfusion in uterine arteries.

The same authors, Sladkevicius et al. [6] in another study say that the measurement of endometrial thickness with transvaginal ultrasonography is a better method for discriminating pathologies within endometrium than that Doppler velocimetry of the uterine arteries. Szpurek et al. [7] presented a different opinion. While evaluating TAM$\mathrm{XV}$ values in uterine arteries in 58 patients with endometrial carcinoma, they showed significantly higher values of this parameter in comparison with TAMXV values in 75 women with no changes detected in the histopathological analysis of the endometrium.

Table 2 Comparison of MEDV $(\mathrm{cm} / \mathrm{s})$ values in the cancer and proliferation groups and in the control group

\begin{tabular}{lccccrrrr}
\hline & $\begin{array}{l}\text { Number } \\
\text { of patients }\end{array}$ & Average & Minimum & Maximum & Median & 25 percentile & 75 percentile \\
\hline Carcinoma & 43 & 10.60930 & 2.800000 & 23.500000 & 10.00000 & 7.600000 & 12.10000 \\
Proliferation & 11 & 8.58182 & 4.800000 & 11.300000 & 8.900000 & 7.600000 & 9.00000 \\
Control group & 46 & 8.99783 & 2.400000 & 21.100000 & 7.950000 & 6.500000 & 11.40000 \\
Total & 100 & 9.64500 & 2.400000 & 23.500000 & 8.900000 & 6.900000 & 11.80000 \\
$P$ value & & $>0.05$ & $>0.05$ & $>0.05$ & $>0.05$ & $>0.05$ & $>0.05$ \\
\hline
\end{tabular}


Table 3 Comparison of PSV $(\mathrm{cm} / \mathrm{s})$ values in the cancer and proliferation groups and in the control group

\begin{tabular}{lccccccc}
\hline & $\begin{array}{l}\text { Number } \\
\text { of patients }\end{array}$ & Average & Minimum & Maximum & Median & 25 percentile & 75 percentile \\
\hline Carcinoma & 43 & 34.11860 & 16.60000 & 55.30000 & 34.10000 & 26.30000 & 39.80000 \\
Proliferation & 11 & 33.61818 & 21.30000 & 48.90000 & 34.200000 & 28.10000 & 36.40000 \\
Control group & 46 & 31.48696 & 13.10000 & 56.70000 & 29.55000 & 23.20000 & 39.80000 \\
Total & 100 & 32.85300 & 13.10000 & 56.70000 & 33.20000 & 25.25000 & 38.40000 \\
$P$ value & & $>0.05$ & $>0.05$ & $>0.05$ & $>0.05$ & $>0.05$ & $>0.05$ \\
\hline
\end{tabular}

In data published in 2005, Englert-Golon et al. [8] also demonstrated the utility of TAMXV in evaluating uterine arteries in women with abnormal postmenopausal bleeding. After analyzing the results of 256 patients, these authors obtained significantly higher values of this parameter in the group of endometrial carcinoma in comparison with the group of proliferations of the endometrium.

In the databases available online, there are few information related to the evaluation of the maximum velocity of blood flow in the uterine arteries. However, after analysis of available data, it can be noted that the authors have reported an increase in the value of these parameters in patients with pathology in the endometrium but such differences were not always statistically significant.

\section{Conclusions}

1. Transvaginal ultrasonography diagnostics using the Doppler technique is a useful method for diagnosing pathologies within the endometrium.

2. Evaluating blood flow velocity in uterine arteries in women with abnormal bleeding from the genitals does not allow groups with a higher risk of proliferation or carcinoma of the endometrium to be distinguished.

Conflict of interest The authors declare that they have no conflict of interest.

Open Access This article is distributed under the terms of the Creative Commons Attribution Noncommercial License which permits any noncommercial use, distribution, and reproduction in any medium, provided the original author(s) and source are credited.

\section{References}

1. Epstein E, Skoog L, Isberg PE et al (2002) An algorithm including results of gray-scale and power Doppler ultrasound examination to predict endometrial malignancy in women with postmenopausal bleeding. Ultrasound Obstet Gynecol 4(20):370-376

2. Lynch PM (1999) Clinical challenges in management of familial adenomatous polyposis and hereditary non polyposis colorectal cancer. Cancer 86(11):25-33

3. Stachowicz N, Czekierdowski A, Daniłoś J, Kotarski J (2002) Use of three-dimensional ultrasonography in the evaluation of the volume of the endometrium in women experiencing bleeding from the uterine cavity. Ginekol Pol 11(73):970-975

4. Warming L, Ran P, Skouby S, Christiansen C (2002) Measurement precision and normal range of endometrial thickness in a postmenopausal population by transvaginal ultrasounds. Ultrasound Obstet Gynecol 5(20):492-495

5. Sladkevicius P, Valentin L, Marsal K (1995) Transvaginal grayscale and Doppler ultrasound examinations of the uterus and ovaries in healthy postmenopausal women. Ultrasound Obstet Gynecol 6(2):81-90

6. Sladkevicius P, Valentin L, Marsal K (1994) Endometrial thickness and Doppler velocimetry of the uterine arteries as discriminators of endometrial status in women with postmenopausal bleeding: a comparative study. Am J Obstet Gynecol 171(3):722728

7. Szpurek D, Moszyński R, Sajdak S, Roszak A (2000) Evaluation of blood flow in the arteries of the uterus in patients with endometrial carcinoma by means of vaginal ultrasonography using the color Doppler technique. Nowotwory 50(1):35-38

8. Englert-Golon M, Szpurek D, Moszyński R, Pawlak M, Sajdak S (2005) Diagnostic value of evaluating blood flow by means of the "power" angio Doppler technique in uterine arteries and in endometrium vessels in women with postmenopausal bleeding. Ginek Pol 76(Suppl 2):134 